\title{
Preparation and Properties of Monodisperse Latex Spheres with Controlled Magnetic Moment for Field-Induced Colloidal Crystallization and (Dipolar) Chain Formation
}

\author{
S. Sacanna and A. P. Philipse* \\ Van't Hoff Laboratory for Physical and Colloid Chemistry, Debye Institute, Utrecht University, \\ Padualaan 8, 3584 CH Utrecht, The Netherlands
}

Received June 8, 2006. In Final Form: September 14, 2006

\begin{abstract}
We report the preparation and properties of monodisperse magnetic poly(methyl methacrylate) latex spheres that exhibit field-induced colloidal crystallization to exotic morphologies controlled by the geometry of the gradient. The magnetic moment of the novel magnetic spheres is due to an inner core of magnetite particles. These particles, obtained from a conventional ferrofluid, first form a monodisperse emulsion with a silane coupling agent, after which they are directly incorporated in PMMA latex synthesized by standard emulsion polymerization. Scattering from the latex shell dominates over light absorption by the magnetic cores such that visible Bragg reflections of the magnetic crystals can be clearly observed. Concentrated nearly white latex fluids may exhibit near a magnet the warped equilibrium menisci known from the usually dark magnetite ferrofluids. Of the many possible applications, we briefly discuss the subsequent growth and melting of crystals by a slowly oscillating gradient, the formation of radial lattices, and the formation of ordered magnetic dots in PMMA latex films.
\end{abstract}

\section{Introduction}

Phase behavior in soft matter involves weak energies comparable to the thermal energy $k T$ which makes crystallization and glass transitions very susceptible to external fields. Spaceshuttle experiments, for example, have demonstrated that the uncharged colloidal spheres which on earth easily form a hardsphere glass state under micro-gravity conditions readily crystallize to periodic structures. ${ }^{1}$ Gravity, on the other hand, may also assist crystallization as in the gravitational settling of colloids into concentrated sediments, sufficiently slow for crystals to nucleate and grow. The gravity field, it should be noted, offers little possibilities for external control of crystallization and to tune the shape, size, and lattice structure of colloidal crystals. Clearly, a magnetic field is much more versatile because of the variety in strength and spatial structure of this field, created either by permanent magnets or electromagnets, in comparison to the fixed homogeneous gravity field. The magnetic option, however, requires colloids which are somewhat exotic: they should be sufficiently monodisperse to crystallize, be sufficiently magnetic to respond to the field-gradient of a small magnet, and have a particle size and optical properties such that any Braggreflections can be visually observed.

Classical emulsion and miniemulsion polymerization in the presence of magnetic nanoparticles may yield latex spheres with magnetic inclusions, but with little control on the amount of magnetic material per particle. ${ }^{2-4}$ Moreover, those methods often require large quantities of surfactants which for many applications (for instance in biomedicine) are undesirable and therefore need to be removed in a later stage. ${ }^{5}$ For this reason, most studies so

* Corresponding author. E-mail: a.p.philipse@chem.uu.nl.

(1) Cheng, Z.; Chaikin, P. M.; Russel, W. B.; Meyer, W. V.; Zhu, J.; Rogers, R. B.; Ottewill, R. H. Mater. Des. 2001, 22, 529-534.

(2) Xu, X. L.; Majetich, S. A.; Asher, S. A. J. Am. Chem. Soc. 2002, 1386413868.

(3) Xu, X. L.; Friedman, G.; Humfeld, K. D.; Majetich, S. A.; Asher, S. A. Chem. Mater. 2002, 14, 1249-1256.

(4) Xu, Z. Z.; Wang, C.; Yang, W.; Deng, Y.; Fu, S. J. Magn. Magn. Mater. 2004, 277, 136-143.

(5) Landfester, K.; Ramirez, L. P. J. Phys.: Condens. Matter 2003, 15, 13451361 far on preparation of magnetic-latex colloids involve coupling reagents, very often silane coupling agents or two-steps swelling techniques. ${ }^{7}$ Coupling reagents usually require multistep procedures to modify the surface of magnetic particles (and/or substrates), such that they can be adsorbed on pre-synthesized seed particles, and eventually can be coated by a latex shell. ${ }^{6,8}$ Swelling techniques, in which magnetic particles are precipitated inside swollen latex, are less time-consuming than the method just mentioned. However, these techniques are only applicable to preparation of large colloids with homogeneous distribution of the magnetic material into the latex matrix. ${ }^{7}$ Moreover, most of the existing methods require a laborious fractionation to obtain a magnetically monodisperse systems, and even when fractionated, those particles still have randomly distributed magnetic material instead of a centered magnetic core.

Though this paper focuses on latex colloids, we note for completeness that, of course, also inorganic colloids such as silica spheres may be employed as carriers for magnetic particles. Earlier work ${ }^{9,10}$ describes silica spheres with a small amount of embedded magnetite particles. Though a magnetic field gradient appeared to have some effect on colloidal crystallization, ${ }^{10}$ the small magnetic moment of these silica spheres is problematic. Replacing the magnetite core by a cobalt ferrite particle enhances the response of silica spheres to a magnetic field ${ }^{11}$ though the magnetic interaction between the spheres is still small, namely of order $10^{-2} \mathrm{kT}$.

Here we report on a novel approach to the synthesis of magnetic latex colloids, which involves the formation of polymerizable magnetite-stabilized emulsions to obtain monodisperse coreshell particles. The magnetic emulsions are used as seed dispersion

(6) Gu, S.; Shiratori, T.; Konno, M. Colloid Polym. Sci. 2003, 281, 1076.

(7) Lindlar, B.; Boldt, M.; Eiden-Assmann, S.; Maret, G. Adv. Mater. 2002, $14,1656-1658$

(8) Huang, Z. B.; Tang, F. Q. J. Colloid Interface Sci. 2004, 275, 142-147. (9) Philipse, A.; van Bruggen, M.; Pathmamanoharan, C. Langmuir 1994, 10 92-99.

(10) Donselaar, L. Silica-Magnetite colloids and magnetic fluids. Thesis, Utrecht University, 1998

(11) Wagner, J.; Autenrieth, T.; Hempelmann, R. J. Magn. Magn. Mater. 2002, 252, 4-6. 
in a standard latex emulsion polymerization. We show that the magnetic latex particles so obtained easily crystallize in a magnetic field gradient, to form three-dimensional bulk crystals with unusual morphologies and microstructures.

The latex spheres are (super)paramagnetic due to a core of magnetite $\mathrm{Fe}_{3} \mathrm{O}_{4}$ nanoparticles. These latter particles, forming the basis of most ferrofluids, are fairly polydisperse, irregular particles that cannot form ordered, crystalline structures. Magnetite, however, can also be made monodisperse, ${ }^{12}$ but still such particles are much too small for visible Bragg reflections. These reflections, it should be noted, would anyhow be difficult to observe since magnetite fluids strongly adsorb visible wavelengths. So it is quite convenient to have a magnetite-based core surrounded by a latex shell which suppresses light absorption and at the same time keeps the cores at distances required for visible Bragg reflections.

Since our preparation method allows a control of the latexshell thickness as well as the magnetic coresize, we can adjust the dipolar attraction between magnetized spheres at contact. Therefore, we have also investigated the latex spheres in a homogeneous field to assess whether chain formation will occur below a critical shell thickness.

\section{Materials and Methods}

A. Preparation. Magnetite Singlets. Aqueous dispersions of magnetite singlets (hereafter referred to as $\mathrm{m}$-particles) were prepared by a coprecipitation described in detail by Massart. ${ }^{13}$ Briefly, an aqueous solution of hydrochloric acid $(10 \mathrm{~mL}, 2 \mathrm{M})$ containing $\mathrm{FeCl}_{2}(2 \mathrm{M})$ was mixed with $40 \mathrm{~mL}$ of $\mathrm{FeCl}_{3}$ aqueous solution (1 $\mathrm{M})$ and afterward added to $500 \mathrm{~mL}$ of ammonia $(0.7 \mathrm{M})$. The obtained magnetite particles could be easily sedimented by placing a magnet under the reaction vessel. The magnetite precipitate was then redispersed in $50 \mathrm{~mL}$ of tetramethylammonium hydroxide $(1 \mathrm{M})$ and allowed to repeptize overnight to obtain an alkaline ferrofluid. Finally, the m-particles were transferred to de-mineralized water by magnetic sedimentation and redispersion.

Magnetite-TPM Emulsions. The m-particles were treated with TPM (3-methacryloxypropyl trimethoxysilane, used as received from Acros Organic) as follows. A dispersion of m-particles $(100 \mathrm{~mL}, c$ $=1.2 \mathrm{~g} / \mathrm{L}$ ) was mixed with $200 \mu \mathrm{L}$ of TPM and gently stirred. The treatment with TPM leads to a particle-stabilized emulsion, hereafter referred to as a m-TPM emulsion. The stability of this emulsion, discussed in detail elsewhere, ${ }^{14}$ is very convenient because the droplets do not coalesce (Figure 1a) such that each initial droplet eventually forms a core for latex polymerization. The m-TPM emulsion droplets can be polymerized to stable, fairly monodisperse composite magnetite-TPM particles (see Figure 1a) when potassium persulfate KPS $(0.4 \mathrm{mM})$ is added and the temperature is raised to $70{ }^{\circ} \mathrm{C}$. An interesting feature of the m-TPM emulsion droplets is that they can be easily polymerized without changing their shape, size, or colloidal stability, as further discussed in section III.

Magnetic PMMA Latex. Polymethyl methacrylate (PMMA) latex was prepared according to a standard soap-free emulsion polymerization. ${ }^{15}$ The essential (and only) modification is the addition of $\mathrm{m}$-TPM emulsion to the starting solution of monomers and initiator. In this paper, we discuss three types of magnetic latex spheres, which only differ in the thickness of the latex shell relative to the magnetic core size. The core-shell colloids are coded as MLx, where $x=1,2$, and 3 denotes a sequence of increasing shell thickness (see Table 1). As a representative example, we describe here the synthesis of ML2 spheres. Magnetic lattices ML2 were prepared by adding $50 \mathrm{~mL}$ of freshly prepared $\mathrm{m}$-TPM emulsion and $8 \mathrm{~mL}$ of

(12) Klokkenburg, M.; Vonk, C.; Claesson, E. M.; Meeldijk, J. D.; Erne, B. H.; Philipse, A. P. J. Am Chem. Soc. 2004, 126, 16706-16707.

(13) Massart, R. IEEE Trans. Magn. 1981, Mag-17.

(14) Sacanna, S.; Kegel, W. K.; Philipse, A. P. Submitted 2006.

(15) Koenderink, G. H.; Sacanna, S.; Pathmamanoharan, C.; Rasa, M.; Philipse, A. P. Langmuir 2001, 17, 6086-6093.

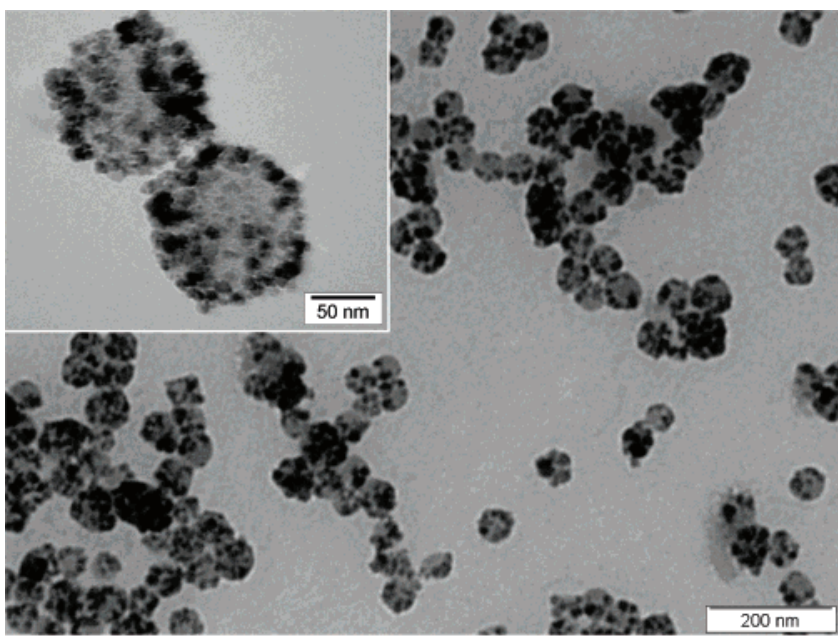

(a)

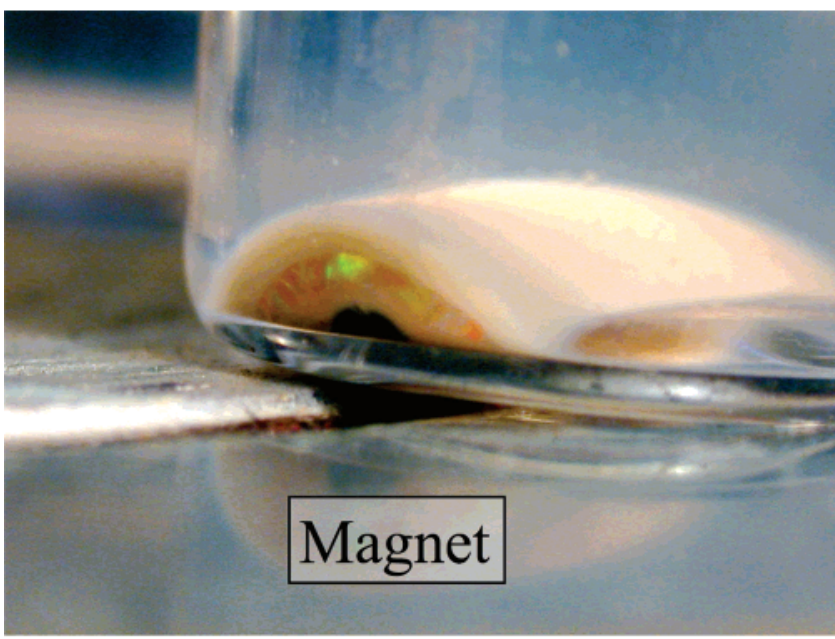

(b)

Figure 1. (a) EM picture of a polymerized m-TPM emulsion imaged by conventional TEM on a dried sample. The insert shows a zoomin of two unpolymerized emulsion droplets taken by Cryo-TEM. (b) Photograph of a magnetically concentrated m-TPM emulsion. Near the magnet the emulsion clearly shows Bragg colors, indicating a crystal-like packing of the droplets, and excluding a possible dropdrop coalescence.

Table 1. Properties of Magnetic Latex Spheres

\begin{tabular}{|c|c|c|c|c|c|c|c|c|}
\hline \multirow[b]{3}{*}{ code } & \multicolumn{4}{|c|}{ TEM } & \multirow{2}{*}{\multicolumn{2}{|c|}{$\begin{array}{c}\text { light } \\
\text { scattering }\end{array}$}} & \multirow{3}{*}{$\frac{\text { mobility }}{\mu \mathrm{m} \mathrm{cm}}$} & \multirow{3}{*}{ 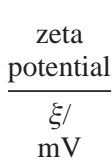 } \\
\hline & \multicolumn{2}{|c|}{ core } & \multicolumn{2}{|c|}{ core-shell } & & & & \\
\hline & $\begin{array}{l}d^{\mathrm{a}} / \\
\mathrm{nm}\end{array}$ & $\begin{array}{l}\sigma / \\
\%\end{array}$ & $\begin{array}{l}d^{\mathrm{b}} / \\
\mathrm{nm}\end{array}$ & $\begin{array}{l}\sigma / \\
\%\end{array}$ & $\begin{array}{l}d^{c /} \\
\mathrm{nm}\end{array}$ & $\begin{array}{l}d^{\mathrm{d} /} \\
\mathrm{nm}\end{array}$ & & \\
\hline $\mathrm{ML}_{1}$ & 94 & & 128 & 6.0 & 192 & 204 & -1.5 & -20 \\
\hline $\mathrm{ML}_{2}$ & 72 & 22 & 210 & 7.8 & 282 & 300 & -1.8 & -24 \\
\hline $\mathrm{ML}_{3}$ & 44 & 19 & 201 & 6.5 & 270 & 300 & -3.9 & -52 \\
\hline
\end{tabular}

${ }^{a}$ Number average diameter with relative standard deviation $\sigma .{ }^{b}$ Total diameter of magnetite-latex spheres. ${ }^{c}$ Diameter from static light scattering. ${ }^{d}$ Diameter from dynamic light scattering. ${ }^{e}$ Zeta potential calculated using Smoluchowsk's equation.

methyl methacrylate to $300 \mathrm{~mL}$ of doubly distilled water (the final magnetite content was about $0.2 \mathrm{~g} / \mathrm{L}$ ) in a $500 \mathrm{~mL}$ round-bottom flask, equipped with a mechanical stirrer and nitrogen inlet. The monomer was emulsified for $1 \mathrm{~h}$ by stirring during which the flask was heated to $70{ }^{\circ} \mathrm{C}$ by immersion into a thermostated oil bath. The polymerization was initiated by injection of $17.5 \mathrm{~mL}$ of KPS solution $(8.5 \mathrm{mM})$ after which the mixture was stirred under nitrogen for 8 $\mathrm{h}$ to ensure complete polymerization (gravimetric analysis confirmed a 93\% of MM conversion). Properties of the magnetic latex spheres 
are given in Table 1. Note that the magnetic core sizes differ because they were prepared from various ferrofluids and different (freshly prepared) m-TPM emulsions. With respect to the magnetite content of the cores, it turns out that elemental analysis of ML2 corresponds to an $\mathrm{Fe}_{3} \mathrm{O}_{4}$ weight \% of 1.7. On the basis of synthesis composition and EM pictures, this weight fraction is expected to be a typical one.

B. Characterization. Electron Microscopy. Transmission electron microscopy (TEM) was performed by using a Philips TECNAI 12 microscope operating typically at $120 \mathrm{kV}$. TEM samples were prepared by drying a drop of diluted dispersion on a glow-discharged copper grid. For scanning electron microscopy (Philips XLFEG30, SEM), grids were prepared as described for TEM but in addition coated with a $7 \mathrm{~nm}$ thick layer of Pt/Pd. Dried colloidal crystals were directly glued to the SEM sample holders. Cryogenic microscopy (cryo-TEM) was employed to directly image m-TPM emulsions in a liquid film glassified by a rapid temperature quench in liquid ethane. Vitrified films were made from m-TPM emulsions on grids coated with holey carbon coating (hole diameter $2 \mu \mathrm{m}$ ). Further information on sample preparation and cryo-TEM imaging of colloids can be found elsewhere. ${ }^{16,17}$ The effect of a homogeneous field for TEM and SEM samples was studied by drying grids in a homogeneous $1.8 \mathrm{~T}$ field between the poles of an electromagnet (Bruker). Samples were dried and exposed together to the magnetic field under exactly the same conditions.

Light Scattering. Static light scattering (SLS) was performed at $25^{\circ} \mathrm{C}$ using an automated setup that scans the angle-dependent scattering intensity produced by a dilute dust free dispersion illuminated by light $(\lambda=546 \mathrm{~nm}$ ) from a mercury lamp (Oriel, mod. 66003). For small values of wave vector $K$ and sphere radius $R$ such that $K R \leq 1$, the Guinier approximation holds: ${ }^{18}$

$$
P(K)=\exp \left(-\frac{K^{2} R_{\mathrm{g}}{ }^{2}}{3}\right)
$$

Thus from the initial slope of the Guinier plot $\left(\ln [I(K)]\right.$ vs $\left.K^{2}\right)$, one obtains the radius of gyration $R_{\mathrm{g}}$ which for a homogeneous sphere yields the particle radius $R_{\mathrm{SLS}}$ via

$$
R_{\mathrm{g}}^{2}=\left(\frac{3}{5}\right) R_{\mathrm{SLS}}^{2}
$$

Dynamic light scattering (DLS) was performed at $25^{\circ} \mathrm{C}$ using an argon laser source operating at $514.5 \mathrm{~nm}$, using the same samples as for SLS. DLS intensity autocorrelation functions were fitted to the second-order cumulant expression $\operatorname{lng}_{\mathrm{I}}(K, t)=\beta_{1}+\beta_{2} t+\beta_{3} t^{2}$ with fitting parameters $\beta_{1}, \beta_{2}$, and $\beta_{3}$. Hydrodynamic radii $R_{\mathrm{DLS}}$ were determined from measured diffusion coefficients using the Stokes-Einstein relation.

Magnetic Properties. Magnetization curves were obtained at room temperature employing an alternating gradient magnetometer $(\mathrm{Mi}-$ croMag 2900 AGM, Princeton Measurements Corp.). Diluted colloidal dispersions (magnetite content typically below 1 vol \%) were contained in small glass cups with internal dimensions of $4 \times$ $3 \times 0.4 \mathrm{~mm}$, whereas dried samples were placed directly on the probe between two pieces of plastic Scotch tape. The saturation magnetization and the diamagnetic susceptibility were obtained by fitting the magnetization curves at high fields (between $1.0 \times 10^{5}$ and $1.2 \times 10^{6} \mathrm{Am}^{-1}$ ) with the Langevin function and an extra term for the diamagnetic contribution

$$
M(H)=M_{\mathrm{s}}\left[\operatorname{coth}(\alpha)-\frac{1}{\alpha}\right]+\chi_{\mathrm{dia}} H ; \alpha=\frac{\mu_{0} m H}{k T}
$$

where $M_{\mathrm{s}}$ is the saturation magnetization of the sample, $\chi_{\text {dia }}$ is the diamagnetic susceptibility, $H$ is the applied magnetic field, $m$ is the magnetic moment per particle, $\mu_{0}$ is the permeability of vacuum, and

(16) Donselaar, L. N.; Frederik, P. M.; Bomans, P.; Buining, P. A.; Humbel, B. M.; Philipse, A. P. J. Magn. Magn. Mater. 1999, 201, 58-61.

(17) Butter, K.; Bomans, P. H. H.; Frederik, P. M.; Vroege, G. J.; Philipse, A. P. Nat. Mater. 2003, 2, 88-91.

(18) Kerker, M. The Scattering of Light; Academic Press: New York, 1969. $k T$ is the thermal energy. The initial susceptibility $\chi_{\mathrm{i}}=$ $(\mathrm{d} M / \mathrm{d} H)_{H \rightarrow 0}$ was determined by a linear fit of the low field data (below $10^{3} \mathrm{Am}^{-1}$ ). The resulting values for $M_{\mathrm{s}}$ and $\chi_{\mathrm{i}}$ were used to calculate the average magnetic particle diameter $d_{\mathrm{M}}$ from the lowfield approximation $(\alpha \ll 1)$ of eq 3

$$
d_{\mathrm{M}}^{3}=\frac{\chi_{\mathrm{i}}-\chi_{\mathrm{dia}}}{M_{\mathrm{s}}} \frac{18 k T}{\mu_{0} M_{\mathrm{s}, \mathrm{bulk}} \pi}
$$

where $M_{\mathrm{s}, \text { bulk }}=4.88 \times 10^{5} \mathrm{Am}^{-1}$ is the saturation magnetization of bulk $\left(\mathrm{Fe}_{3} \mathrm{O}_{4}\right)$

Electrophoresis. Electrophoretic mobilities were determined (Coulter DELSA $440 \mathrm{SX}$ ) for dilute samples at a $\mathrm{pH}$ of 6 , a temperature of $25^{\circ} \mathrm{C}$, and an ionic strength of $20 \mathrm{mM} \mathrm{NaCl}$ (i.e., a Debye-Hückel screening length of $\kappa^{-1}=2 \mathrm{~nm}$ ). Zeta potentials (Table 1) were calculated from mobilities using Smoluchowski's equation which is valid here since particle sizes are large compared to the Debye screening length. ${ }^{19}$

Elemental Analysis. The magnetite weight fraction was calculated from the iron weight fraction (ICP elemental analysis performed at Department of Earth Sciences, Utrecht University), assuming all iron is present as $\mathrm{Fe}_{3} \mathrm{O}_{4}$.

Mass Densities. The particles mass densities $\rho_{\mathrm{p}}$ were obtained from dispersion mass densities measured with a density meter (AntonPaar DMA5000) thermostated at $T=25.000{ }^{\circ} \mathrm{C}$.

Infrared Spectroscopy. Transmittance infrared (IR) spectra for m-TPM emulsions were collected using a PerkinElmer Spectrum 2000 FTIR spectrometer. IR samples were prepared by adding 280 $\mathrm{g}$ of $\mathrm{KBr}$ to a known amount of dispersion and drying the mixtures at $100{ }^{\circ} \mathrm{C}$ for $12 \mathrm{~h}$ in open air. The obtained powders were pressed into $1 \mathrm{~mm}$ thick disks (diameter $=13 \mathrm{~mm}$ ).

Colloidal Crystallization. The effect of a field gradient on the formation and morphology of colloidal crystals was qualitatively investigated using permanent (often $\mathrm{NdFeB}$ ) magnets of various shapes and geometries as further discussed in section III. Samples were left undisturbed on a heavy marble table in a thermostated room for periods varying from days to several months. After exposure to a field gradient, samples could always be easily redispersed, and no flocculation was visible during or after this exposure. Growth rate and morphology of crystal structures were as judged by eye fairly reproducible. Gravitational settling occurred only significantly on a time scale of at least several weeks, whereas field-induced crystallization was in most cases visible within few hours. In the absence of a magnetic field gradient, crystallization required a centrifuge. For example, ML3 dispersion produced overnight a Braggreflecting crystalline sediment when centrifuged at $1000 \mathrm{rpm}$ in a (Beckmann) table centrifuge.

\section{Results and Discussion}

A. Preparation. Our main result is a novel and convenient method for preparing monodisperse magnetic latex spheres with a magnetizable core and adjustable PMMA latex shell. Instead of chemically modifying single magnetic particles, we let them self-assemble at the oil-water interface of TPM emulsion droplets (Figure 1a) which leads to magnetite clusters with controlled size.

An m-TPM emulsion can be easily prepared by gently stirring the right amount of TPM with a water-based magnetite dispersion. No turbulent mixing is needed as usual is the case in emulsion preparation or oil drop extrusion through a membrane as in the work of Shang et al. ${ }^{20}$ The m-TPM emulsion is rather insensitive to temperature, and it remains stable whether prepared at room temperature or at $80{ }^{\circ} \mathrm{C}$. Moreover, due to the adsorbed $\mathrm{m}$-particles, the emulsion droplets can be concentrated and sedimented by an external magnetic field. In some cases, quite

(19) Henry, D. C. Proc. R. Soc. London, Ser. A 1931, 133, 106.

(20) Shang, H.; Chang, W. S.; Kan, S.; Majetich, S. A.; Lee, G. U. Langmuir 2006, 22, 2516-2522. 


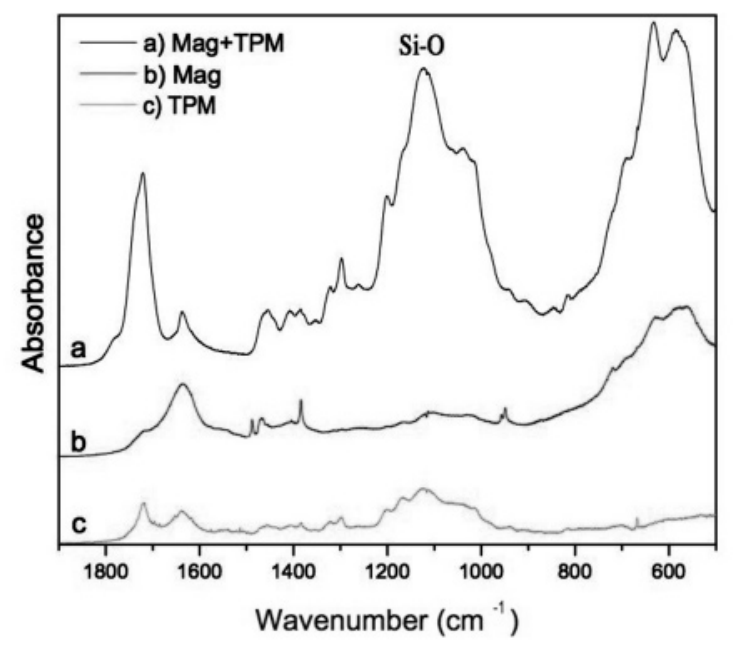

(a)

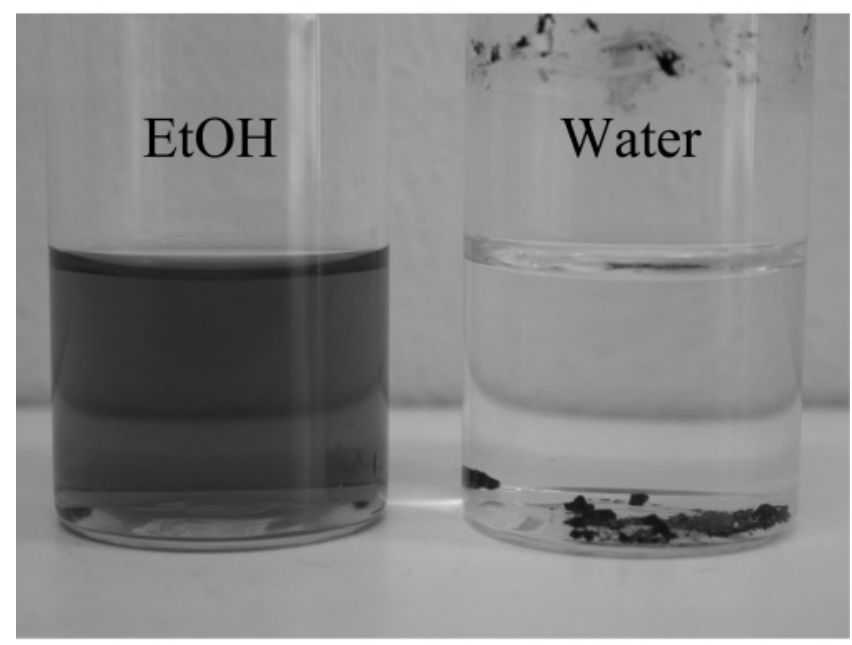

(b)

Figure 2. (a) IR spectra of TPM, magnetite, and m-TPM emulsion magnetite. After being washed with ethanol, the magnetite coming from the m-TPM emulsion shows a strong absorption peak at about $1100 \mathrm{~cm}^{-1}$ indicating that some TPM is still adsorbed at the magnetite surface. (b) When $\mathrm{KBr}[1 \mathrm{M}]$ is added to a m-TPM emulsion the magnetic droplets coalesce forming the hydrophobic aggregated visible in the right sample. Those aggregates easily redisperse in pure ethanol giving stable suspensions of TPM coated magnetite particles (left sample).

surprisingly, Bragg colors were observed in magnetically concentrated m-TPM emulsions (see Figure 1b) confirming that the emulsion droplets do not coalesce and remain monodisperse even when pressed together by an external force.

The m-TPM emulsions clearly form an interesting system in itself, since salt addition may destabilize the emulsion and the resulting hydrophobic flocs can be easily redispersed in ethanol giving stable dispersions. Figure $2 \mathrm{~b}$ shows a coalesced emulsion in an aqueous $1 \mathrm{M} \mathrm{KBr}$ solution, and a stable dispersion of $\mathrm{m}$-particles obtained by redispersing the flocs in ethanol. In ethanol, we would expect the TPM to be completely dissolved, but peaks around $1100 \mathrm{~cm}^{-1}$ in the IR spectra of Figure 2a (corresponding to the $\mathrm{Si}-\mathrm{O}$ modes from the TPM) prove that some TPM is still present on the magnetite surface even after washing the particles with ethanol. The presence of adsorbed TPM explains the particle stability in ethanol due to steric repulsions. Moreover, the resulting hydrophobic surface of the magnetite, due to the methacrylate groups of the adsorbed TPM, induces flocculation of the m-particles in aqueous solutions, in

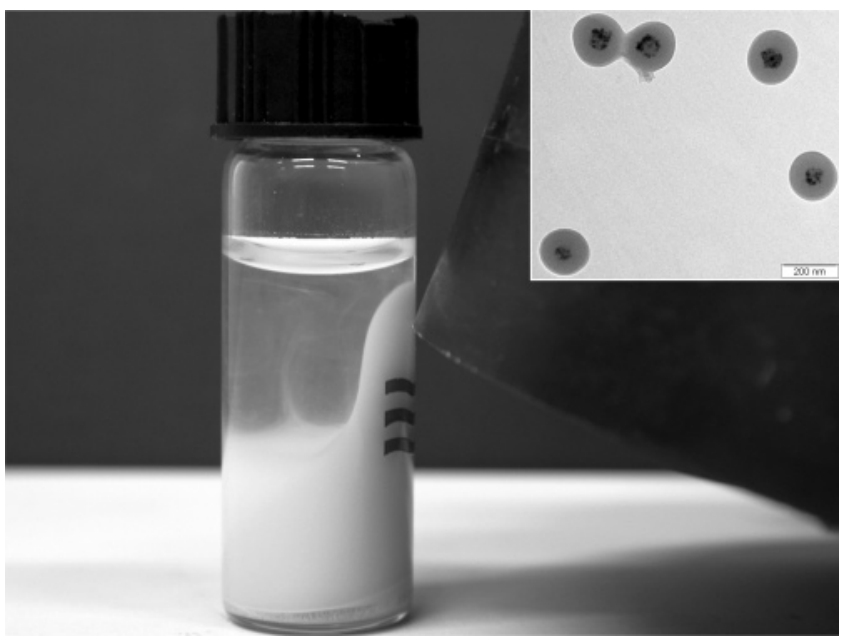

Figure 3. ML3 dispersion exhibits, near a magnet, a deformed equilibrium meniscus. In contrast to the usual dark-brown magnetite ferrofluids, this system is nearly white due to the light scattered from the PMMA shell. The insert shows a TEM view of ML3 spheres.

marked contrast to the initial situation in which the magnetite particles are present in the form of a stable m-TPM emulsion.

The m-TPM emulsion apparently is not affected by the presence of MMA, added before the polymerization. The mixture remains unchanged until the initiator is injected and then rapidly turns turbid. Electron microscopy and light scattering analysis shows no evidence of any homogeneous nucleation of the PMMA latex: it always polymerizes directly onto the seeds presented by the m-TPM emulsion. These seeds are already fairly monodisperse and further growth always reduces the polydispersity ${ }^{21}$ which explains the narrow size distribution of the final magnetic latex spheres. Their polydispersity $(5-6 \%)$ is comparable to commercial calibration latex spheres (PolyScience, Inc.) of similar average size. Just as in the case of a conventional emulsion polymerization, ${ }^{22}$ the final particle size can be controlled quite precisely by changing, within certain limits, the initial monomer concentration or by repeated seeded growth on the same system. Depending on how thick the latex shell grows on the magnetic cores, the color of the dispersion changes from dark brown to almost white. Since secondary latex nucleation is virtually absent and all individual magnetite clusters are encapsulated by a latex shell, there is no need for purification procedures or additional fractionation, which contributes to a fairly high yield. In all cases, we only purified reaction mixtures by one single sedimentation and redispersion step. Here we also note that we were able to reproduce the synthesis up to a $1.5 \mathrm{~L}$ scale without significant differences in terms of particles size and polydispersity.

B. Properties. Visual Observations. The samples color varies depending on the latex shell thickness. In ML3, where the latex shell is $78 \mathrm{~nm}$, the light absorption from the $\mathrm{Fe}_{3} \mathrm{O}_{4}$ core is dominated by light scattering from the latex shell, which gives rise to visible Bragg reflections from the magnetic crystals (see Figures 4, 5, and 13). Figure 3 shows a nearly white ML3 dispersion, which close to a permanent magnet exhibits the warped (equilibrium) meniscus which so far only seems to have been observed for concentrated, dark magnetite ferrofluids. ${ }^{23}$

Electron Microscopy. Figure 6 shows a SEM picture of a dried crystal of ML2 spheres. The particles are slightly deformed

(21) Philipse, A. P. In Fundamentals of Interface and Colloid Science; Lyklema, J., Ed.; Elsevier: Amsterdam, 2005; Vol. IV.

(22) Fitch, R. M. Polym. React. Eng. 2003, 11, 911-953.

(23) Rosensweig, R. E.; Elborai, S.; Lee, S.; Zahn, M. J. Magn. Magn. Mater 2005, 289, 192-195. 


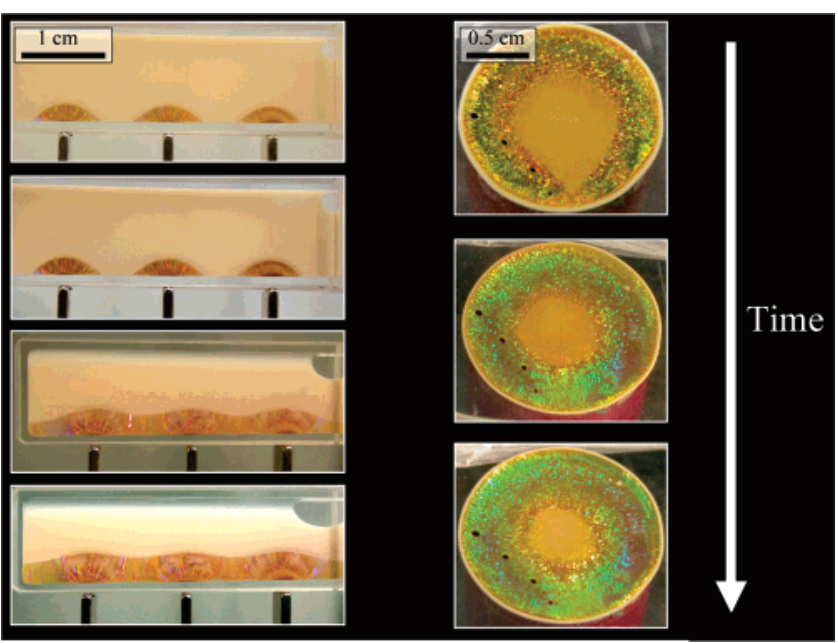

Figure 4. Photograph time series showing a magnetic field induced crystal growth with two different spatial field geometries for ML3 spheres. Depending on the size of the magnetic core, the formation of those bulky crystals takes hours or days, whereas in the absence of a magnetic field, crystallization due to gravitational settling takes several weeks.

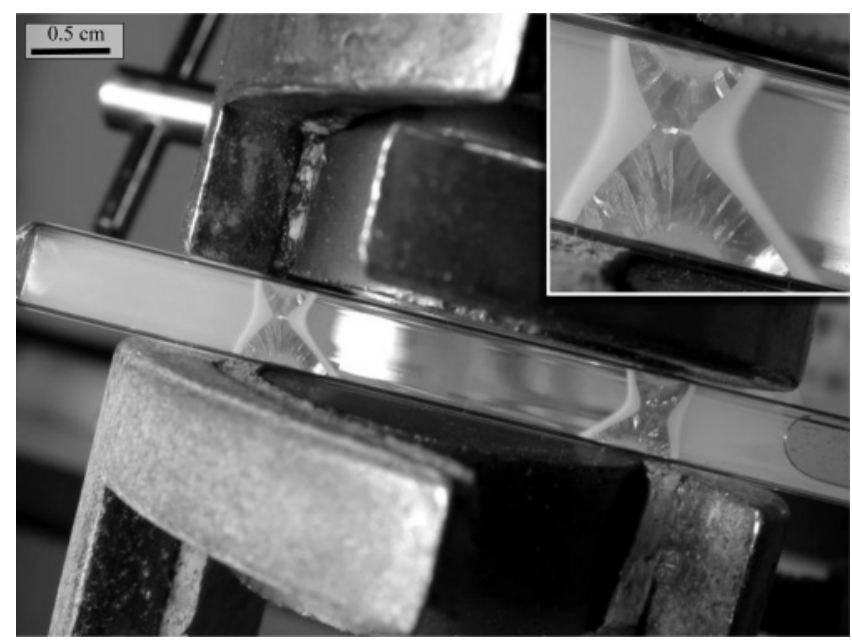

Figure 5. When a capillary tube, containing an initially homogeneous magnetic latex dispersion, is placed between the poles $(\mathrm{N}-\mathrm{N})$ of two permanent magnets, the particles rapidly migrate and crystallize according to the local magnetic field gradient maximum. The picture shows the resulting magnetic crystals after $3 \mathrm{~h}$ of exposure to the magnetic field.

because of the fast degradation of the latex due to the electron beam exposure. In the insert, a TEM picture shows a single ML2 particle. Due to the difference in contrast between latex and iron oxide, the particle core shell structure is clearly visible. Like we observed for SEM imaging, the severe conditions in TEM analysis easily damages the latex particles shell, which results in measured TEM diameters of about $30 \%$ smaller than the light scattering values (see Table 1). DLS measures hydrodynamic diameters which are usually larger than (hard sphere) diameters detected by TEM. However, this difference is typically on the order of only a few percent, and in our experiments, it is largely offset by the shrinkage due to particles degradation. This shrinkage is not related to the core-shell morphology: we observed a very similar shrinkage for pure PMMA spheres. TEM pictures also demonstrate the importance of using a m-TPM emulsion as starting seed dispersion in order to achieve a well-defined coreshell particle structure with monodisperse cores. An interesting consequence of the magnetic core in each latex sphere is the possibility to create an ordered two-dimensional array of magnetic

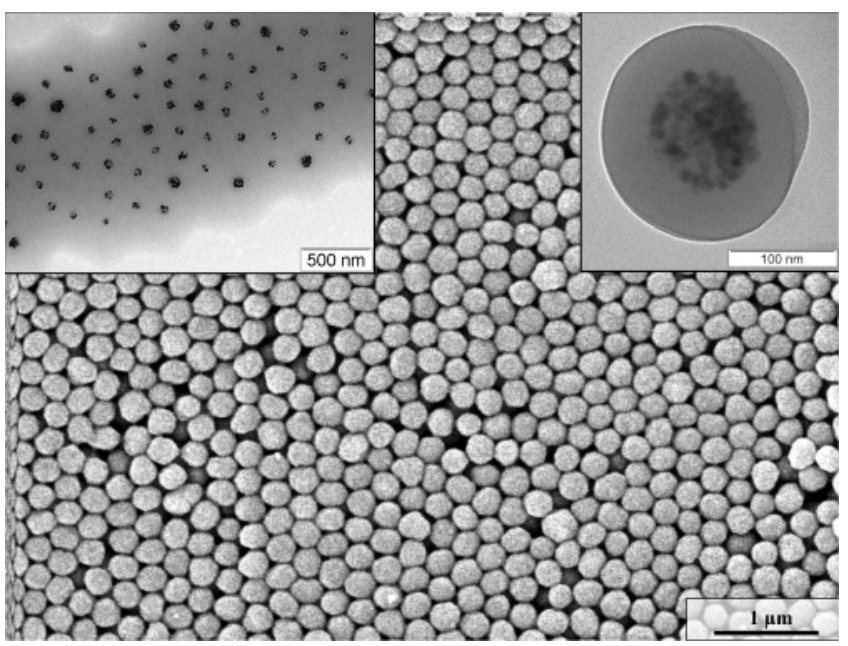

Figure 6. SEM micrograph of a dry magnetic colloidal crystal (ML3 spheres). In the inserts, (right) TEM image of one single colloid shows the core-shell particle structure and (left) latex film with ordered magnetic dots, resulting after melting the latex shell.

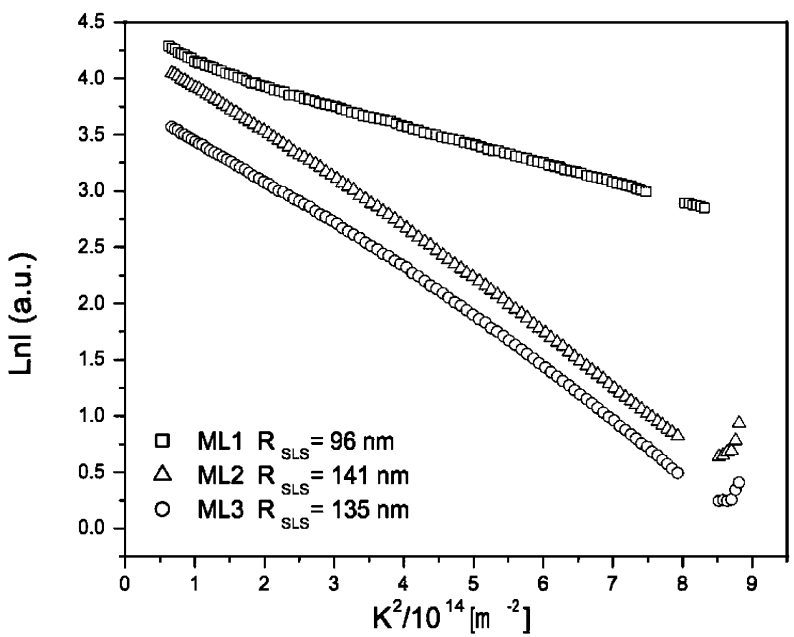

Figure 7. Guinier plots and calculated radii for ML1, ML2, and ML3. For KR1, $\ln I(K)$ versus $K^{2}$ is a straight line as expected from eq 1 .

particles in a latex film. When the latex of a $2 \mathrm{D}$ crystalline structure melts in an electron beam, the m-TPM cores protrude a latex film at distances equal to the latex sphere diameter (Figure 6).

Cryogenic electron microscopy allows a direct in situ imaging of m-TPM emulsions in a vitrified solvent, giving a realistic view on the particle-stabilized droplets in the emulsion. However, since the TPM molecule has a vinyl end group it is possible to polymerize the emulsion droplets by addition of a radical initiator like KPS, and image the resulting composite magnetite-polyTPM particles with conventional TEM as well. Figure 1a shows a TEM picture of a polymerized emulsion and in the insert a CryoTEM view of the same emulsion before the polymerization. We observe that the emulsion droplets once polymerized maintain the same spherical shape and a fairly monodisperse size distribution, and the magnetite particles remain on the particle surface.

Light Scattering. Figure 7 shows SLS measurements for the three different systems ML1, ML2, and ML3. In all cases, $\ln (I)$ depends linearly on $K^{2}$ as expected from eq 1 . The SLS radii (Table 1) are in good agreement with the radii determinate by DLS (Figure 8), confirming the low polydispersity observed by electron microscopy. In some cases, light scattering measurements were repeated at different wavelengths (typically at $\lambda=365$, 


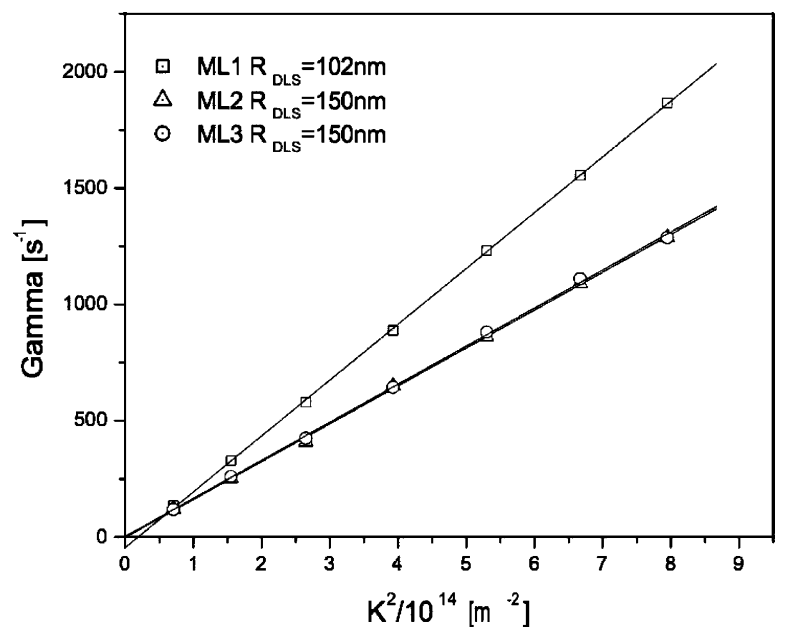

Figure 8. Decay rate of the electric field autocorrelation functions measured with DLS at various wavevectors $K$, for ML1, ML2, and ML3 spheres.

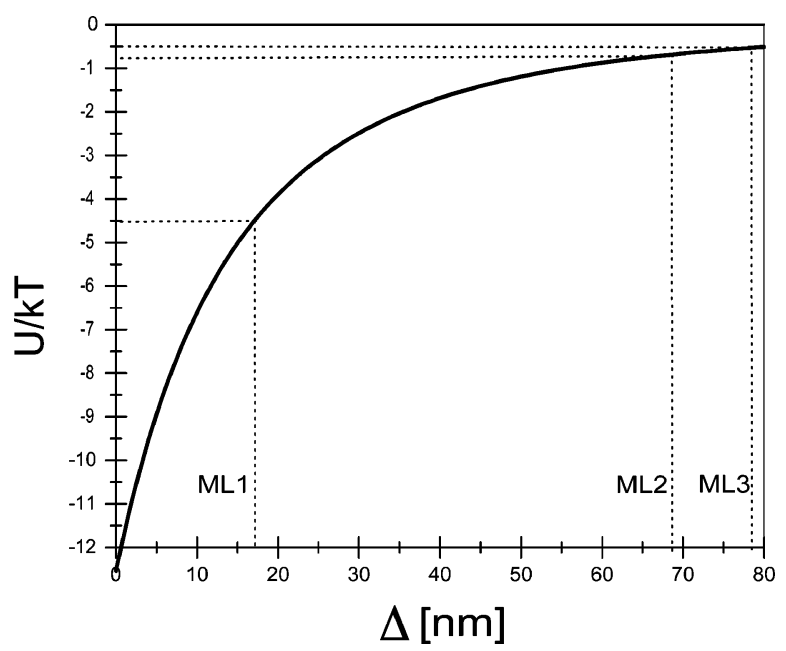

Figure 9. Dipolar interaction potential between two magnetic latex particles at contact as a function of the latex shell thickness $\Delta$. The curve has been calculated for ML2 particles, and references to ML1 and ML3 in the plot are just indicative.

546 , and $578 \mathrm{~nm}$ ) giving comparable results which are in complete agreement with SLS data for pure PMMA spheres. Therefore, we conclude that the magnetic core is also optically buried into the latex: even for the thin latex shell sample ML1, the core does not affect light scattering measurements.

Electrophoresis. Electrophoretic mobilities of the magnetic latex particles are reported in Table 1, together with zeta potentials obtained using the Smoluchowski approximation. The spheres have negative zeta potentials and their magnitude increases with the latex shell thickness. This could be explained by considering that the corresponding negative surface charge is mainly due to the acidic sulfate $\left[\mathrm{R}-\mathrm{OSO}_{3}\right.$ - $]$ end-groups generated in the MMA polymer by the radical initiator KPS. Since the magnetite particle cores at neutral $\mathrm{pH}$ are close to the isoelectric point of magnetite $\left(\mathrm{pH}_{\mathrm{iep}}=7\right)$, they will carry little charge so the main contribution to the particles charge will be due to the latex shell. ML2 and ML3 particles, for example, have the same size $(d=300 \mathrm{~nm})$ but different cores, and this leads to a zeta potential of magnitude $28 \mathrm{mV}$ higher for ML3 which have a significantly smaller core $\left(\mathrm{ML} 3 d_{\mathrm{CORE}}=44 \mathrm{~nm}\right.$; ML2 $\left.d_{\mathrm{CORE}}=72 \mathrm{~nm}\right)$.

Magnetic Properties. Magnetization measurements on the magnetic latex dispersions indicate the absence of a permanent particle magnetic moment, excluding a possible magnetic dipole

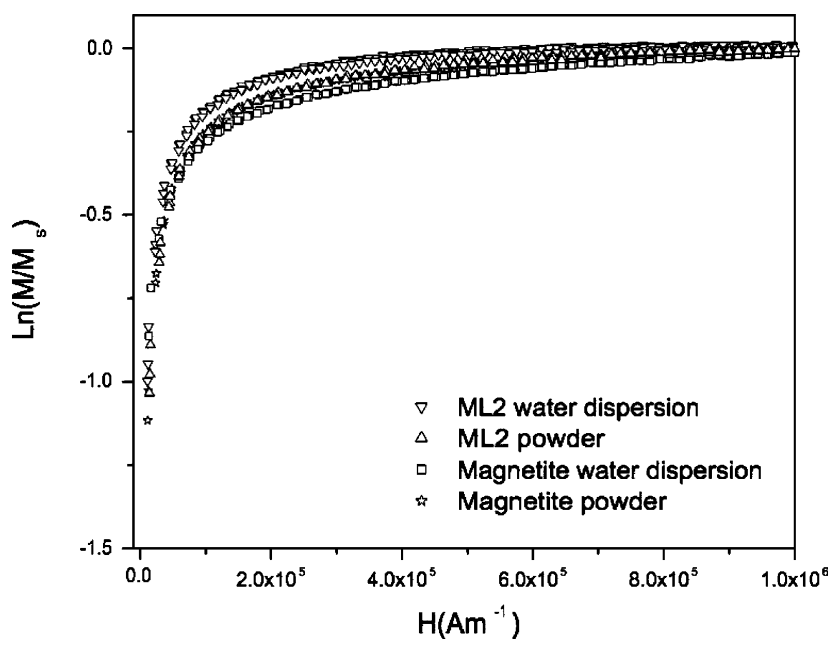

Figure 10. Magnetization measurements on ML2 and magnetite, showing the logarithm of the normalized magnetization as a function of the applied magnetic field.

Table 2. Magnetite Diameter Determined by TEM and Magnetization Measurements on Dierent Samples

\begin{tabular}{|c|c|c|c|c|c|c|}
\hline \multirow[b]{2}{*}{ code } & \multicolumn{2}{|c|}{ magnetic diameter $^{a}$} & \multicolumn{2}{|c|}{$\mathrm{MR} / \mathrm{MS}^{b}$} & \multicolumn{2}{|c|}{ TEM $^{c}$} \\
\hline & $\begin{array}{l}\text { disp. } \\
\text { [nm] }\end{array}$ & $\begin{array}{l}\text { pwd } \\
{[\mathrm{nm}]}\end{array}$ & $\begin{array}{c}\text { disp. } \\
{[\%]}\end{array}$ & $\begin{array}{l}\text { pwd } \\
{[\%]}\end{array}$ & {$[\mathrm{nm}]$} & {$[\sigma \%]$} \\
\hline magnet & 14 & 11 & 4 & 2 & 11 & 28 \\
\hline ML2 & 12 & 11 & 4 & 3 & & \\
\hline
\end{tabular}

${ }^{a}$ Magnetic diameter from magnetization measurements. ${ }^{b}$ Remanence/ saturation magnetization ratio. ${ }^{c}$ TEM number-averaged diameter $(1000$ counts).

coupling from the magnetite singlets into the lattices. Figure 10 shows the logarithm of the normalized magnetization curves for magnetic latex, magnetite samples in aqueous dispersion, and dried magnetite powder. The overlap between the curves, and the lack of significant hysteresis and remanence, confirm that magnetic latex and magnetite, either dried or dispersed in water behave as typical superparamagnetic colloids. Moreover, magnetic diameters calculated from magnetization measurements on the different samples (see Table 2) are all in good agreement with the average magnetite size determined by TEM $\left(d_{\text {TEM }}=11 \mathrm{~nm}\right)$. The total magnetic moment $\mu_{\text {tot }}$ induced by magnetizing the core will therefore be linearly proportional to the number $n$ of $\mathrm{m}$-particles it contains. From elemental analysis on ML2 particles, we estimated an $\mathrm{Fe}_{3} \mathrm{O}_{4}$ weight fraction of about $1.7 \%$. Considering that the measured particle mass density $\rho_{\mathrm{ML} 2}$ is $1.20 \mathrm{~g} \mathrm{~cm}^{-3}$, we calculated $n_{\mathrm{ML} 2}=28$, which looks reasonable in view of TEM pictures (see for instance Figure 6).

With respect to structure formation (section III C), it is relevant to convert the magnetite weight fraction to a dipolar attraction between core-shell colloids as follows: ${ }^{24}$

$$
\frac{U(d)}{k T}=-\frac{\mu_{0} \mu_{\mathrm{tot}}^{2}}{2 \pi k T d^{3}}
$$

where $\mu_{0}=4 \pi \times 10^{-7} \mathrm{H} \mathrm{m}^{-1}$ is the permeability in a vacuum, $\mu_{\text {tot }}$ is the total magnetic moment of a single composite particle, $k T$ is the thermal energy, and $d$ is the center to center distance between two particles. In Figure 9, the interaction potential calculated for ML2 particles is shown as a function of the latex shell thickness $\Delta$.

(24) Claesson, E. M.; Philipse, A. P. Langmuir 2005, 21, 9412-9419. 


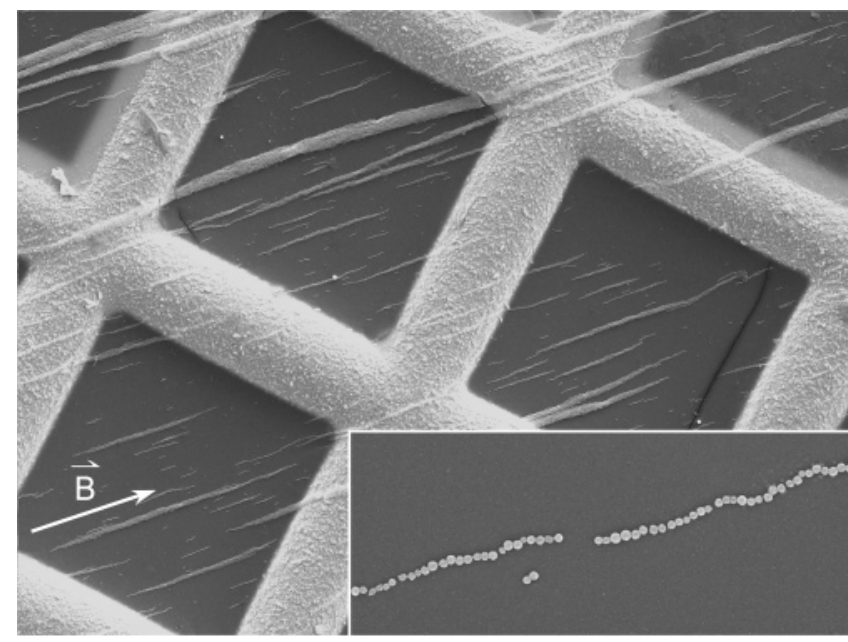

(a)

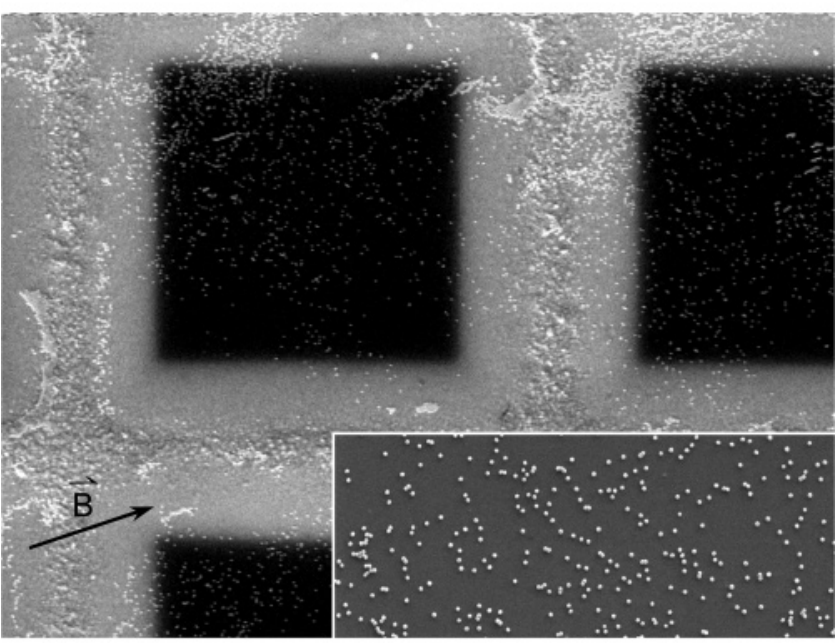

(b)

Figure 11. (a) SEM picture of a ML1 sample exposed to a homogeneous field of $1.8 \mathrm{~T}$, which shows field-oriented chains of particles. (b) ML3 spheres exposed to a homogeneous field of 4.5 T remain randomly distributed on a SEM grid, showing that a large latex shell thickness effectively screens the induced magnetic dipole interaction between particles.

C. Field-Induced Structure Formation. Particle-particle interaction due to the magnetic induced dipole moments can be tuned by changing the latex shell thickness of the particles. For instance, from the interaction potential calculated for the system ML2 (Figure 9), we would expect, in a saturating homogeneous magnetic field, formation of particle chains only for latex shell thickness smaller than $60 \mathrm{~nm}$. This is confirmed by SEM analysis on particles dried under a homogeneous magnetic field. In Figure 11a, ML1 particles, which compared to ML2 have a bigger core $\left(n_{\mathrm{ML} 1}>n_{\mathrm{ML} 2}\right)$ and a thinner latex shell $(\Delta=17 \mathrm{~nm})$, show field-induced chain formation in a homogeneous field of $1.8 \mathrm{~T}$, but ML3 particles, with a smaller core $\left(n_{\mathrm{ML} 3}<n_{\mathrm{ML} 2}\right)$ and a larger shell $(\Delta=78 \mathrm{~nm})$, retain their isotropic distribution even if the field strength is increased up to $4.5 \mathrm{~T}$ (Figure 11b).

Thus, a sufficiently thick latex shell (see also Figure 9) screens almost completely the field-induced magnetic moments of the cores such that even in a magnetic field the net colloid-colloid interaction is purely repulsive. It is well-known ${ }^{25}$ that repulsive spheres may undergo a disorder-order transition from an amorphous fluid to a slightly denser colloidal crystal. To densify

(25) Russel, W. B.; Daville, D. A.; Schowalter, W. R. Colloidal Dispersions; Cambridge University Press: Cambridge, 1991.

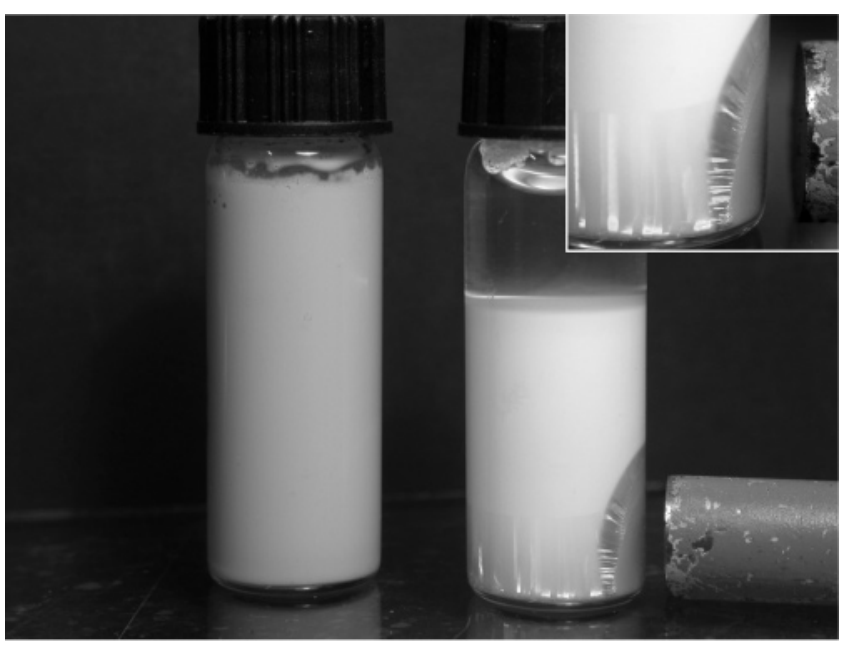

Figure 12. Selective separation in a colloidal binary mixture of magnetic and nonmagnetic latex spheres. On the right bottle, it is also possible to observe how gravity- and magnetic field-induced crystals grow independently in different orientations.

a dispersion for this transition to occur, often particle sedimentation is employed ${ }^{25}$ (though also filtration ${ }^{26}$ and solvent evaporation $^{27}$ has been used). In our case, however, the required densification is easily (and rapidly) achieved by an inhomogeneous magnetic field $\vec{H}$ that exerts a force

$$
F_{\mathrm{m}}=(m \cdot \vec{\nabla}) \vec{H}
$$

on the magnetic moment $m$ of a core. As a result the colloids translate toward a magnet (see, for example, Figure 3) to regions of high field gradient where, consequently, an opposing gradient in osmotic pressure $\Pi$ is set up. In equilibrium, the net force on a colloid is zero

$$
\frac{1}{c} \vec{\nabla} \Pi(c)=(m \cdot \vec{\nabla}) \vec{H}+\frac{4}{3} \pi R^{3} \delta \vec{g}
$$

Here we have also included the gravitational force on a sphere of radius $R$ with mass density $\delta$. The equilibrium colloid concentration $c$ in the inhomogeneous field can, in principle, be calculated from 7 when the equation of state $\Pi(c)$ is known and the field gradient is well-defined and fairly simple (which is not the case, for example, in Figures 3-5). Qualitatively, three regions in a concentration profile may be distinguished: an intermediate region where the density is high enough to drive crystallization but still low enough for the spheres to move to lattice positions, a more concentrated glassy region near a magnet where sphere mobility is too low to relax the amorphous structure to a crystal, and a dilute region far away from a magnet where sphere concentration is too low for crystals to nucleate. The inserts in Figures 5 and 12 show two examples of these three regions, namely a crystalline layer between an amorphous region near the magnet and a dilute dispersion.

Magnetic field gradients can be employed to achieve unusual crystal morphologies. In Figure 4, inhomogeneous fields with two different spatial geometries were used to grow circular and wavelike colloidal crystals. Figure 5 shows a capillary tube containing ML2 dispersion, placed between opposite poles of two magnets. It is possible to observe particles migrating along the tube, attracted to the region of high magnetic field gradient, and eventually form crystals in the local magnetic field gradient

(26) Philipse, A. P.; Pathmamanoharan, C. J. Colloid Interface Sci. 1993, 159 , 96-107.

(27) Philipse, A. P. J. Mater. Sci. Lett. 1989, 8, 1371-1373. 


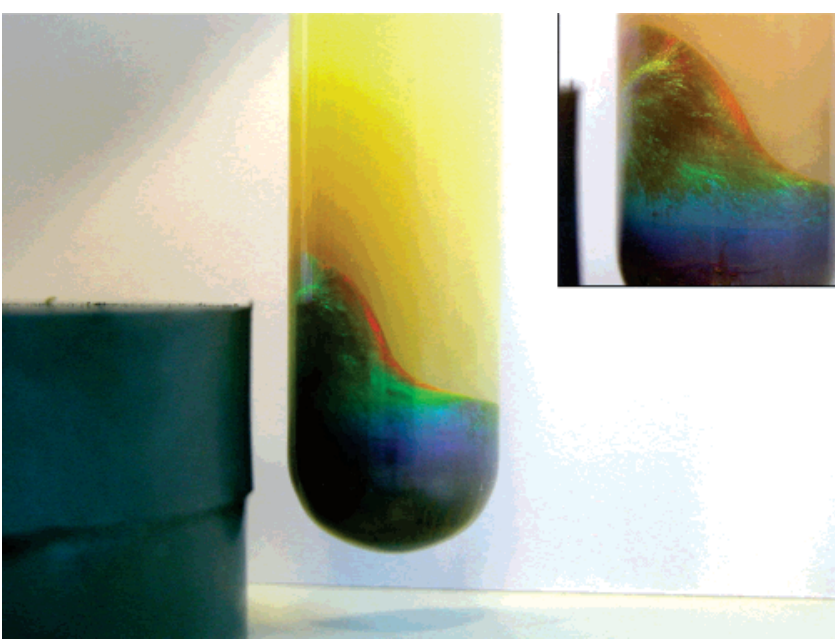

Figure 13. Magnetic crystals exhibiting variations in Bragg colors due to the inhomogeneous field gradient which compresses or relaxes the crystal lattice. The Bragg color pattern also changes in time when the magnet is (manually) oscillated at a frequence on order of $1 \mathrm{~s}^{-1}$.

maximum. This interesting feature can be used for example as physical separation method. In Figure 12 is shown how a binary mixture of pure nonmagnetic, pure PMMA latex and magnetic latex, respectively, 120 and $105 \mathrm{~nm}$ in radius, can be efficiently separated just by placing a small permanent magnet close to the bottle. Moreover, for a binary mixture of magnetic and nonmagnetic particles, we can induce crystal formation by means of the gravitational field and a magnetic field with different spatial orientation into the same sample. In Figure 12, for instance, the magnetic field is oriented at $90^{\circ}$ with respect to the gravitational field, and this causes magnetic and nonmagnetic colloids to crystallize independently from each other.

The color of the Bragg reflected light arising from a colloidal crystal at a certain angle depends on the interparticle distance into the lattice. In a magnetic colloidal crystal, the magnetic field gradient may vary this distance by compressing or relaxing the particle lattice, resulting in a field dependent color of the Bragg light. Asher et al. ${ }^{28}$ measured such influence on crystalline colloidal arrays in low ionic strength aqueous solutions. However, no images or pictures of a "bulk" colloidal magnetic crystal were provided. As shown earlier in this paper, our magnetic latex spheres can easily form such bulky crystals, allowing visual observation of the Bragg color shift cause by a variation in the magnetic field gradient (see Figure 13).

(28) Xu, X. L.; Friedman, G.; Humfeld, K. D.; Majetich, S. A.; Asher, S. A. Adv. Mater. 2001, 13, 1681-1684.

\section{Conclusions and Outlook}

In conclusion, a convenient method has been developed for the preparation of monodisperse PMMA latex core-shell spheres with a magnetizable magnetite core. The crucial step is the formation of a magnetite-stabilized emulsion, comprising monodisperse magnetic cores which can be easily incorporated in latex via surfactant free emulsion polymerization. For sufficiently thick latex shells, the nearly white dispersions form Braggreflecting crystals in a magnetic field gradient with unusual morphologies that cannot be achieved by gravity. For a sufficiently thin latex shell, the colloids form extensive dipolar chains and anisotropic aggregates in a homogeneous field. The possibility to go from strongly magnetizable spheres, where a field induces dominating dipolar attractions, to spheres for which the magnetic core has no effect on interactions offers interesting possibilities. It allows, for example, to study chain formation as a function of induced dipole moment as well as investigating bulk crystal formation of isotropic spheres where the core only serves to increase the concentration by their migration in a field gradient. Our magnetic latex spheres might also be employed in magnetic tweezer experiments ${ }^{29}$ where control of particle size and magnetic moment is important, and for fabricating magnetic latex films with ordered arrays of magnetic nanodots. In addition, functionalization of the latex shell may be of interest in view of the many therapeutic and diagnostic applications of magnetic colloids in pharmacy and medicine. ${ }^{30}$

Acknowledgment. Julian Lopez-Viota and Maria Claesson are thanked for preliminary synthesis work and helpful discussions. We are grateful to Hans Meeldijk for cryo-TEM pictures and Ben Erné for his help on IR and magnetic measurements. Silvia Fazzini is thanked for additional synthesis and characterization work. This work was financially supported by The Netherlands Organization for Scientific Research (NWO/Stichting Chemische Wetenschappen).

Supporting Information Available: Color versions of Figure 3, 5, and 12 are offered. A comparison between the color of ML1, ML2, and ML3 dispersions at similar particle concentration is shown in Figure 14. This material is available free of charge via the Internet at http://pubs.acs.org.

\section{LA0616505}

(29) Koenig, A.; Hebraud, P.; Gosse, C.; Dreyfus, R.; Baudry, J.; Bertrand, E.; Bibette, J. Phys. Rev. Lett. 2005, 95, 128301.

(30) Elaissari, A. Colloidal Biomolecules, Biomaterials and Biomedical Applications; Dekker Marcel: New York, 2004. 